\title{
Characterization of the Impingement Dynamics of Pulsed Rocket Plumes with the Ground at Low Ambient Pressure
}

\author{
Manish Mehta ${ }^{1}$, Nilton O. Renno ${ }^{2}$, Aline J. Cotel ${ }^{3}$ \\ University of Michigan, Ann Arbor, MI 48109 USA \\ and \\ Rob M. Grover III ${ }^{4}$ \\ NASA Jet Propulsion Laboratory \\ California Institute of Technology, Pasadena CA 91109 USA
}

\begin{abstract}
[Abstract] This study focuses on the impingement dynamics of pulsed supersonic plumes with the ground at Mars atmospheric conditions. Experiments show that large transient pressure peaks with average amplitudes between 35 and $42 \mathrm{kPa}$ (4.5-5.2 psi), and durations between 10 and $18 \mathrm{~ms}$ develop at the surface during the impingement of a $10 \mathrm{~Hz}$ pulsed underexpanded jet. These transient pressure peaks are superimposed on "quasi-steady" pressure perturbations with amplitudes of about $5 \mathrm{kPa}(0.87 \mathrm{psi})$ and duration between 35 and $40 \mathrm{~ms}$. This interaction corresponds to a $20 \mathrm{~Hz}$ ground shock frequency. Numerical and experimental data show that the large transient pressure amplitudes are caused by the formation and collapse of plate shocks (normal reflected shocks close to the ground surface) formed during the startup and shutdown cycle of the pulse-modulated thrusters. These pressure perturbations may cause soil liquefaction and gas-soil bursting which can lead to substantial soil erosion. Our goal is to understand the dynamics of pulsed jet impingement during terminal descent and quantify crater formation, dust lifting, and spacecraft stability at low ambient pressure. This study is the first step in this direction.
\end{abstract}

\begin{tabular}{|c|c|c|}
\hline & & Nomenclature \\
\hline$P_{c}$ & $=$ & thruster inlet stagnation pressure \\
\hline$T_{c}$ & $=$ & thruster inlet temperature \\
\hline$P_{a m b}$ & $=$ & ambient pressure \\
\hline$e$ & $=$ & thruster plume expansion ratio \\
\hline$\gamma$ & $=$ & ratio of the specific heat capacities at constant pressure and constant volume \\
\hline$M$ & $=$ & Mach number \\
\hline$D_{e}$ & $=$ & rocket nozzle diameter \\
\hline$h$ & $=$ & height or distance from the ground \\
\hline
\end{tabular}

\section{Introduction}

—UTURE landed missions to Mars and the Moon need reliable and well understood landing systems. Economical - soft landing systems are important to turn the US vision for space exploration into reality [4]. The understanding of the interactions of thruster plumes with the surface, plume/thruster feedbacks caused by these interactions, and the quantification of dust lifting are important for assessments of lander stability and contamination by dust lifted

\footnotetext{
${ }^{1}$ Graduate Student, Dept. of Atmos. \& Space Sciences, 2455 Hayward St, AIAA Member. manishm@umich.edu

${ }^{2}$ Associate Professor, Dept. of Atmos. \& Space Sciences, 2455 Hayward St, AIAA Member

${ }^{3}$ Assistant Professor, Dept. of Civil and Environmental Engineering, 1351 Beal Ave

${ }^{4}$ Lead Engineer, Phoenix Entry, Descent and Landing Systems, MS 301-490
} 
during terminal descent, that is, during the last few seconds before touchdown. Thus, these studies are extremely important not only for the engineering but also for the science of future missions [4].

The entry, descent and landing (EDL) phase is a high-risk area of space missions. This occurs because it is extremely challenging to safely land a spacecraft in a poorly known environment in the short amount of time available for the EDL phase. The understanding of the fluid dynamics of the impingement of rocket plumes on planetary surfaces is very important for the success of future landers. Previous investigations of plume interactions with the surface were conducted mostly by NASA researchers in the 1960s and 70s. These past studies focused on steady-state rocket plumes such as those produced by the Viking and Apollo descent engines [4]. Studies of pulsed rocket plumes such as the ones produced by the Phoenix (PHX) spacecraft engines are virtually non-existent. Phoenix is the first scout mission to Mars, and the first to explore its northern polar region [4]. Today's technology and analysis techniques allow us to easily study pulsed jets and expand on earlier studies of the interaction of steadystate thruster plumes with the ground. There are three main areas of concerns to mission planners related to plumeground interaction: 1. Control authority deterioration; 2. Surface alteration and crater formation; and 3. Dust lifting during landing [4].

Control authority of lander's descent needs to be fully understood, so that it can be accurately represented in Guidance, Navigation and Control (GNC) systems. Ground effect and lift-loss, in the presence of topographical features and jet-induced site-alteration, can cause asymmetric loadings on spacecrafts during terminal descent [4]. This leads to undesirable torques which can perturb the spacecraft. Rigorous GNC models of these processes for pulse-modulated thrusters during terminal descent do not currently exist. In order to minimize the risks to landings, these processes must be understood and incorporated into Lander Simulators (LanderSim/POST) [2]. The impingement of supersonic plumes with the ground can excavate the surface during landing. Once the shear stress due to pressure perturbations caused by plume impingement with the surface exceeds the bearing capacity of the soil, ground failure occurs [4]. This failure can lead to site-alteration, crater formation and the lifting of large quantities of sand and dust. The main reason for concern is that this may lead to lander instability, damage of its underside, and alteration of the surface of scientific interest. Dust lifting and settling on the lander can cause instrument contamination and perhaps failure [4]. This could also decrease the overall power available to the spacecraft because of the settling of lifted dust on solar panels. Investigations of site alteration by supersonic pulsed jets and dust lifting and deposition during spacecraft landings have not been reported in the literature yet.

\section{Experimental Cold Flow Testbed (CFTB)}

We developed an experimental testbed to study the impingement dynamics of supersonic pulsed jets with the surface at Mars ambient pressure (Figure 1a, b). Various quantitative diagnostics have been installed into the CFTB to measure important physical quantities. The quantitative diagnostics are composed of: 1 . Eight Freescale ground pressure sensors (with $1 \mathrm{~ms}$ response time and pressure range between 0 and $53 \mathrm{kPa}$ ); 2. One OMEGA thruster Pc transducer (0-3.44 MPa); 3. One MKS Baratron 627 B absolute pressure sensor (0-700 Pa); 4. Various Analog Devices thermocouples. The ground pressure sensors and thermocouples are placed in a radial fashion along a clear acrylic impingement plate (Figure 1b). The pressure sensors start at the centerline of the impingement plate and are spaced $25 \mathrm{~mm}$ apart until the periphery of the plate. To obtain supersonic flow through the nozzle, a general contour of the MR107 converging-diverging nozzles employed on the Phoenix terminal descent engines have been implemented. The OMEGA Pc transducer is located at the inlet of the engine's thruster chamber. In order to reduce the mass flow and the interactions of the rocket plumes with the chamber walls, a half-scale model of the Phoenix thruster engine is used in our testbed. The thruster chamber stagnation pressure (Pc), pulse width (PW), thrust and motor frequency are controlled to guarantee dynamical similarity with the real rocket plume. Dry compressed nitrogen has been used as the plume gas to simulate the specific heat ratio of hydrazine by-products. Numerical modeling and past studies suggest that the temperature effects are of second order and do not significantly alter the flow. Thus, the current system does not control the plume temperature [5].

The thruster model is inserted into a Mars thermal-vacuum chamber, and the chamber temperature and pressure are monitored. This cold flow testbed system (CFTB) achieved all performance requirements necessary to simulate the thruster plumes of the Phoenix (PHX) pulse-modulated Terminal Descent Engines (TDE) (Table 1). The

performance requirements set by the PHX TDE during the constant velocity descent phase are: $10 \mathrm{~Hz}$ Pulse-Width Modulation (PWM) Frequency; 1.24 MPa maximum Pc, and 151.7 MPa/s Pc-rates during engine startup/shutdown 
cycles. The pressure at the nozzle inlet (Pc) and the ground impingement pressures are transiently monitored with a USB 6009 DAQ unit with a total sampling rate of $48 \mathrm{kHz}$. The distance of the thruster from the surface can be adjusted between 0.5 and $2.2 \mathrm{~m}$. All hardware components have been either fabricated at the University of Michigan or obtained from commercial vendors.

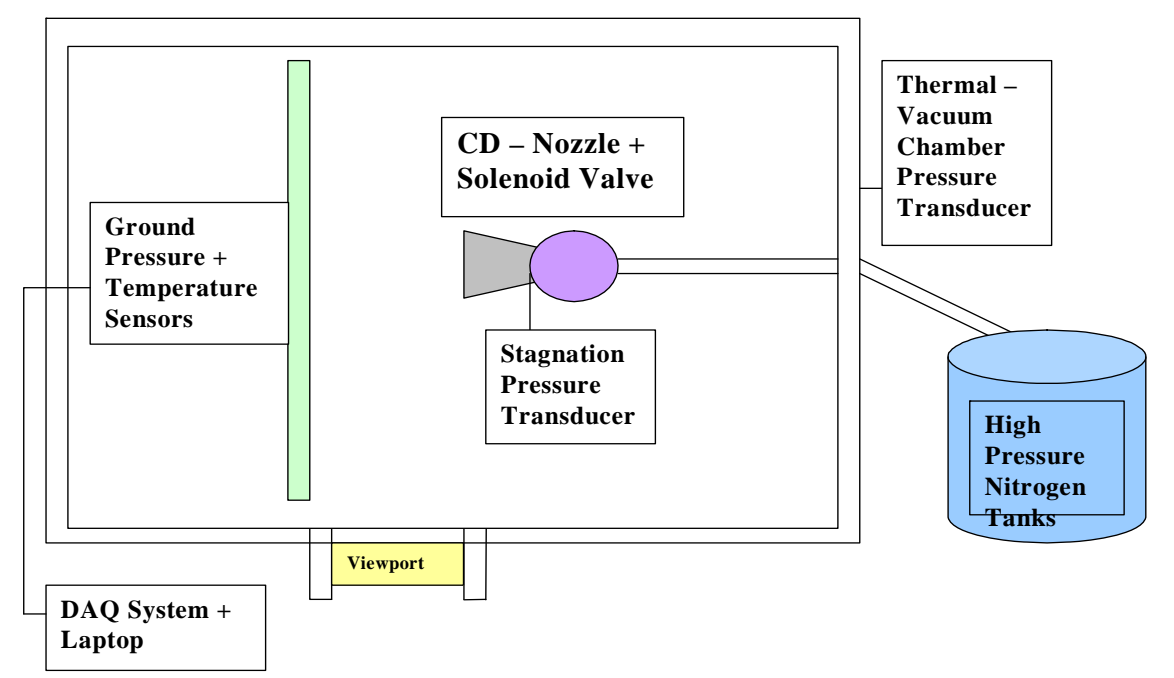

Figure 1a. Schematic of the University of Michigan Cold Flow Testbed system.

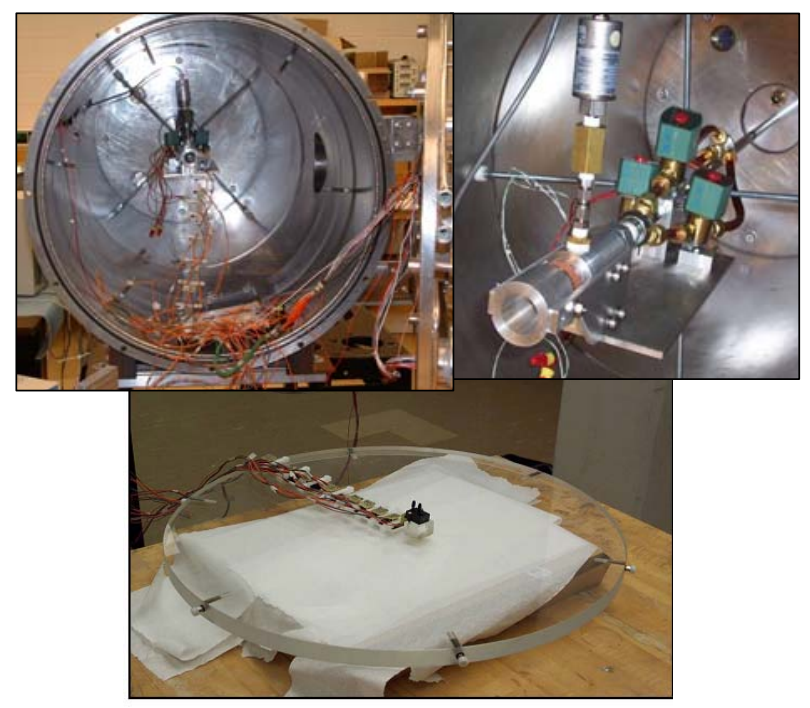

Figure 1b. Images of the Cold Flow Testbed system. 


\section{Thruster Plume’s Scaling Parameters}

Table 1. Important physical parameters

\begin{tabular}{|l|l|l|}
\hline Parameters & Model & $\begin{array}{l}\text { Full- } \\
\text { Scale }\end{array}$ \\
\hline $\begin{array}{l}\text { Hypersonic-Similarity } \\
\text { Parameter }(k)\end{array}$ & 11.3 & $9.9-7.0$ \\
\hline Expansion Ratio (e) & 4.4 & $5.5-3.3$ \\
\hline $\mathrm{P}_{\mathrm{c}}(\mathrm{MPa})$ & 1.24 & 1.24 \\
\hline $\mathrm{T}_{\mathrm{C}}(\mathrm{K})$ & 298 & 1350 \\
\hline Gamma $(\gamma)$ & 1.4 & $\begin{array}{l}1.25 \\
\text { (variable) }\end{array}$ \\
\hline $\begin{array}{l}\text { Pulse Width (ms) @ Max } \\
\text { Pc }\end{array}$ & 45 & 45 \\
\hline $\begin{array}{l}\text { Engine Pulsed Mode } \\
\text { Frequency (Hz) }\end{array}$ & 10 & 10 \\
\hline
\end{tabular}

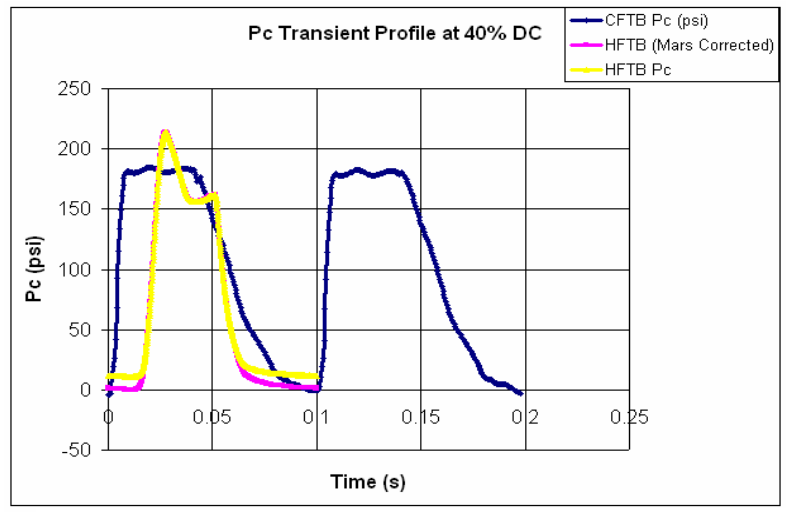

Figure 2. Pc comparison between HFTB and CFTB.

Two main parameters must be matched for the cold gas jet to be dynamically similar to the real thruster plume: the hypersonic similarity parameter (Eq. 1) and the expansion ratio (Eq. 2) [3]. The hypersonic similarity term is the ratio of the rocket plume's kinetic energy with respect to its internal energy. The expansion ratio is directly related to the expansion angle of the plume as it exits the nozzle. A larger expansion ratio leads to a larger expansion of the plume. Transient parameters such as pulse width and pulse frequency also need to be matched to the Phoenix engine specifications. The important parameters that need to be considered for proper scaling are tabulated in Table 1. Temperature effects are of second order because gas temperature does not significantly affect the shock structure [3]. The hypersonic similarity term and expansion ratio were matched between our half-scale test model and the fullscale PHX TDE (Table 1).

$$
\begin{aligned}
& k=\gamma(\gamma-1) M^{2} \\
& e=\frac{P_{e}}{P_{a m b}}
\end{aligned}
$$

\section{Flow Visualization}

A single pass-mirror reflectance shadowgraph imaging system was developed for the visualization of the transient shock structure.

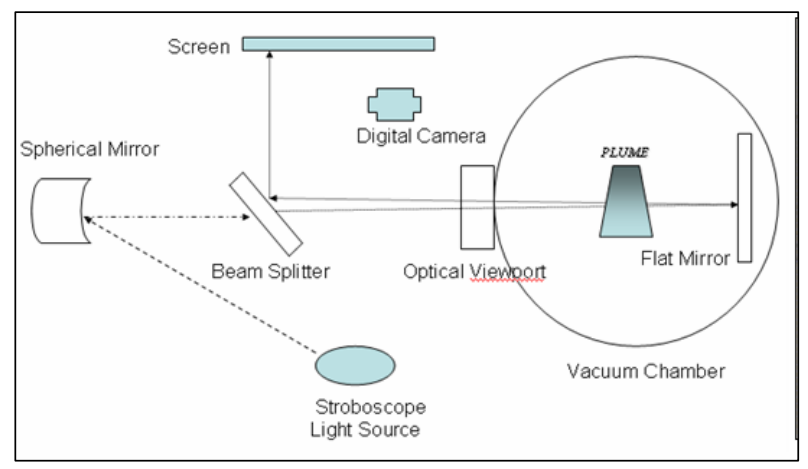

Figure 3. Schematic of single pass shadowgraph imaging system.

A light beam passes through the thermal-vacuum chamber test section, and the beam is reflected off of a flat mirror attached to the interior of the chamber wall. A 1534 Strobotac AB pulses the light beam at a frequency of one $\mathrm{kHz}$ for the visualization of the transient shock structure. A parabolic-mirror collimates the light beam and a 50/50 beam splitter directs the shadowgraph image on a Da-Lite High-contrast screen (Figure 3). The shadowgraph image is 
captured with a 7.0 MPx Nikon Digital camera. A collimated light will soon replace the stroboscope and a highspeed digital camera, capable of taking up to 16000 frames per second, will be used to capture the transient behavior of the plate shock formation and collapse process [7].

\section{CFD Analyses}

Axisymmetric computational fluid dynamics (CFD) models of the pulsed plume-ground interactions at low ambient pressure have been developed in collaboration with Lockheed Martin’s Aerophysics Department (LM). GASP [12] (applied by LM) and FLUENT [13] (applied by the University of Michigan) CFD models were applied to study these complex plume-ground interactions. The University of Michigan simulations were performed at two specific heights: 0.53 meters (PHX touchdown altitude) and 1.25 meters. The CFD models were forced with a time series of the thruster chamber pressure (Pc) measured during tests at the Phoenix hot fire testbed (HFTB). The time series of Pc pressure were obtained by averaging data from twelve engine tests [3]. In addition, Pc time series measured during CFTB experiments were used in a few CFD simulations for comparison with simulation forced by data from the real engine. Most of the numerical simulations done at the University of Michigan were for cold flow cases. Two different FLUENT models were used to perform simulations: A coupled second order implicit inviscid model and a coupled second order implicit k-epsilon turbulent model. Due to significant plate shock instabilities, convergence required a time-step size of $1 \mathrm{e}-5 \mathrm{~s}$.

\section{Results}

\section{A. Pressure Measurements.}

Preliminary cold flow test data of experiments at the Phoenix touchdown altitude $(0.53 \mathrm{~m})$ show two centerline transient ground pressure peaks of average amplitude range between 24 and $42 \mathrm{kPa}$, and duration ranging between 10 and $18 \mathrm{~ms}$ (Figure 4b). This correlates to a ground shock frequency of $20 \mathrm{~Hz}$. A “quasi-steady state" region with ground pressure amplitude of $5 \mathrm{kPa}$ develops for between 35 and $40 \mathrm{~ms}$. Within the quasi-steady state region, minor pressure perturbations with frequencies ranging between 0.5 and $1.8 \mathrm{kHz}$ are observed. These large transient pressure amplitudes are also observed at altitudes of $0.76 \mathrm{~m}$, but with smaller amplitudes. The maximum pressure

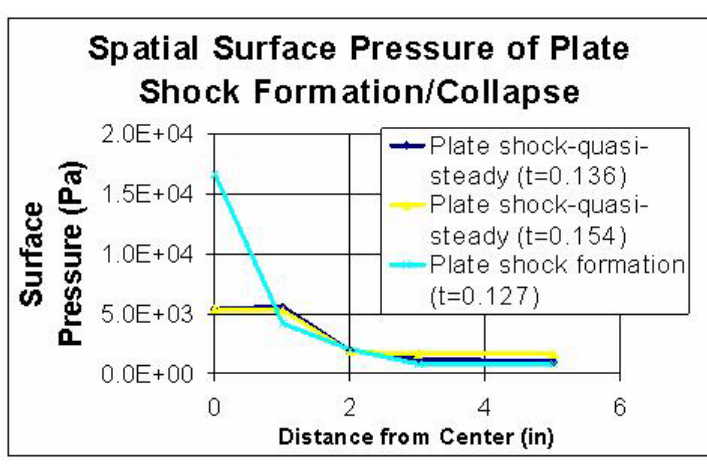

Figure 4a. Experimental spatial pressure profiles at touchdown $(0.5 \mathrm{~m})$.

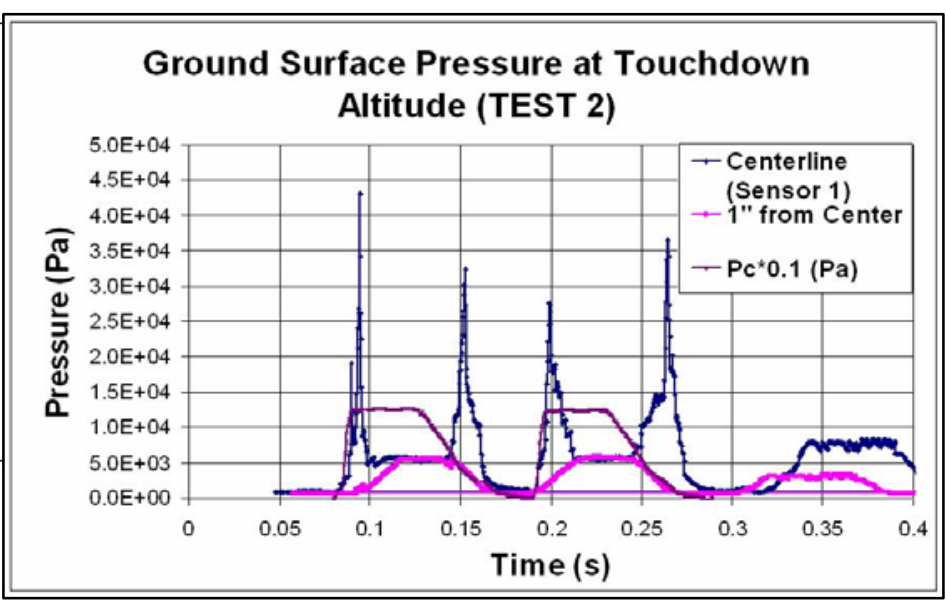

Figure 4b. Experimental ground pressure/Pc temporal profiles at touchdown $(0.5 \mathrm{~m})$.

peaks at this altitude have amplitudes between 22 and $26 \mathrm{kPa}$ with duration of approximately 10-18 ms and the quasi-steady pressure peaks have amplitudes of $4.4 \mathrm{kPa}$ for $40 \mathrm{~ms}$ [5]. Ground pressure profiles were also modeled by CFD simulations, and the results were compared with these experiments (Figure 6). At higher altitudes, the centerline ground pressure at both the pre and post plate shock regimes showed a non-linear behavior (AppendixFigure 9). During plate shock formation, the centerline pressure amplitude is $17 \mathrm{kPa}$ and at quasi-steady state regime, the pressure amplitude below the fully developed plate shock decreases to $5 \mathrm{kPa}$ (Figure 4a). 


\section{B. Flow Visualization Data}

Visualization of the "quasi steady-state" supersonic jets produced by the Phoenix cold flow testbed model was performed during some of our tests (Figure 5). This image was obtained using the single pass-mirror reflectance shadowgraph imaging system described above. However, the visualization system for shock-ground interactions caused by pulsed, under-expanded jets is still being implemented. Our main goal is to visualize the plate shock formation/collapse dynamics seen in the numerical simulations.

Numerical simulations of the transient plume shock structure at $1.25 \mathrm{~m}$ will provide insights on the ground interaction physics.

\section{Discussion}

\section{A. Transient Ground Pressure Profile Analyses}

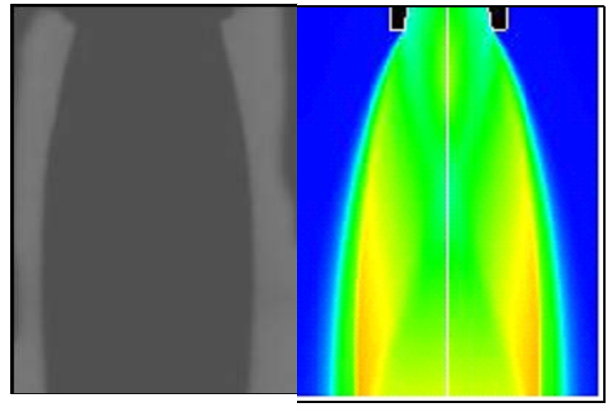

The pressure at the Phoenix thruster chamber (Pc) and its rate of change obtained during HFTB are in good agreement with values obtained by the University of Michigan CFTB, with a PWM of 45 ms (Figure 2). Water-hammering effects at the full-scale engine valves produce an asymmetric double pressure peak at $50 \mathrm{~ms}$ in the data from HFTB testing [2]. During cold flow testing, water hammer effects were negligible. This leads to a slight disparity between the data from these two experiments (Figure 2).

CFD simulations and laboratory experiments show that large non-linear pressure perturbations with frequencies of about 20

Figure 5. Shadowgraph (left) and CFD (right) near-field exhaust plume comparison - cold flow tests. $\mathrm{Hz}$ develop at the ground, below the plate shock region, during the 10 $\mathrm{Hz}$ engine startup and shutdown portions of the duty cycle. Minor pressure oscillations on the order of $1 \mathrm{kHz}$ also develop during the quasi-steady phase of the cycle. These oscillations may partially be due to Hartman's fundamental modes [8].The amplitude of the large pressure perturbations are on the order of 5 percent (42 kPa) of the stagnation pressure at the inlet thruster chamber (Pc), and depend on the stagnation pressure rise/fall rates, the ambient conditions (atmospheric density), and the strength of the plate shock (distance of the spacecraft from the surface). The CFD simulations and laboratory experiments were conducted for spacecraft altitudes landing from $1.8 \mathrm{~m}$ above the surface to touchdown. We believe that the large pressure overshoots observed are caused by plate shock instabilities during its' formation and collapse.

The plate shock dynamics is described next. At the start of the engine duty cycle, isentropic compression waves propagate downward from the converging-diverging nozzle and results in direct flow impingement on the ground. This causes compression of the flow and the pressure significantly increases at the ground. An irreversible fully developed reflected shock forms when the compression waves coalesce at a particular standoff distance from the ground. This causes the ground pressure to suddenly decrease because of turbulent and viscous dissipation at the shock boundary [3]. Due to the stable plate shock, the dynamic pressure component significantly decreases. Prior to the formation of a developed plate shock, a weak, unstable, convex-shaped reflected shock forms and the stand-off distance increases with increasing nozzle exit pressure (Figure 7b). A similar process occurs during plate shock collapse. During the engine shutdown phase, the compression waves weaken and cannot sustain a normal reflected shock because of the decrease in the nozzle exit pressure. Upon shock collapse, there is a surface pressure increase because of the direct flow impingement at the ground (Figure 7a). Post shock collapse causes the weak convex shock structure to move closer to the ground plane (Figure 7a). As the nozzle exit pressure decreases further, the compression wave strength decreases and causes the surface pressure to drop. Upon impingement, the flow expands along the surface at high velocities or what is known as a wall jet [3]. When the plate shock is within the quasisteady regime, the ground pressure for our application is at approximately $5 \mathrm{kPa}$, an approximate $4 \mathrm{kPa}$ increase from the ambient conditions. This mechanism contributes strongly to the large transient pressure overshoots observed during the engine startup and shutdown phase of a pulse-modulated thruster.

Additional ground pressure perturbations are caused by instabilities at the boundaries of a stagnation bubble and plate shock that forms during the quasi-steady portion of the duty cycle. The underexpanded oblique 
shocks within the plume flow field interact with the plate shock and this leads to a triple shock structure (Figure 7b). The ensuing slip-line impingement leads to a local increase in ground pressure near the outskirt of the plate shock compared to the centerline pressure. This tends to form a stagnation bubble underneath the reflected normal shock. Plate shock fluctuations normal to the surface due to stagnation bubble formation and collapse contribute to small ground pressure perturbations [1].

Our CFD simulations show that the maximum diameter of the plate shock is approximately $1.5 \mathrm{D}_{\mathrm{e}}$ at nozzle height of $0.53 \mathrm{~m}$. The plate shock is a slightly curved structure at a standoff distance of $1.2 \mathrm{D}_{\mathrm{e}}$ at touchdown altitude (Figure $7 \mathrm{a}, \mathrm{b}$ ). The spreading of these transient pressure peaks may be related to the Pc rise/fall times. The flow physics in the impingement zone is extremely unsteady and additional studies are underway to understand these processes in more detail [7]. From this study, we conclude that there is good agreement between CFD and experimental data of the dynamics of cold flow impingement at the ground (Figure 6). These results also correlate very well with respect to numerical simulations of axisymmetric hot gas pulsed jet impingement at Mars ambient pressure [7].

Preliminary studies of steady state under-expanded jets, under similar forcing and boundary conditions, show that the ground pressure is approximately constant with time, with only minor oscillations of amplitude +/$0.0025 \%$ of Pc. No transient pressure spikes were noticed during tests and CFD simulations of steady-state flow impingement.

\section{B. Spatial Ground Pressure Profile Analyses}

The spatial pressure profiles at the centerline and the point $25 \mathrm{~mm}$ adjacent to it show different pressure profiles during normal shock formation and the quasi-steady state regimes (Figure 4b). This same behavior is observed during plate shock collapse. A characteristic decrease in ground pressure is observed after shock formation. It can be seen through these experiments that the plate shock diameter is less than $50 \mathrm{~mm}$.

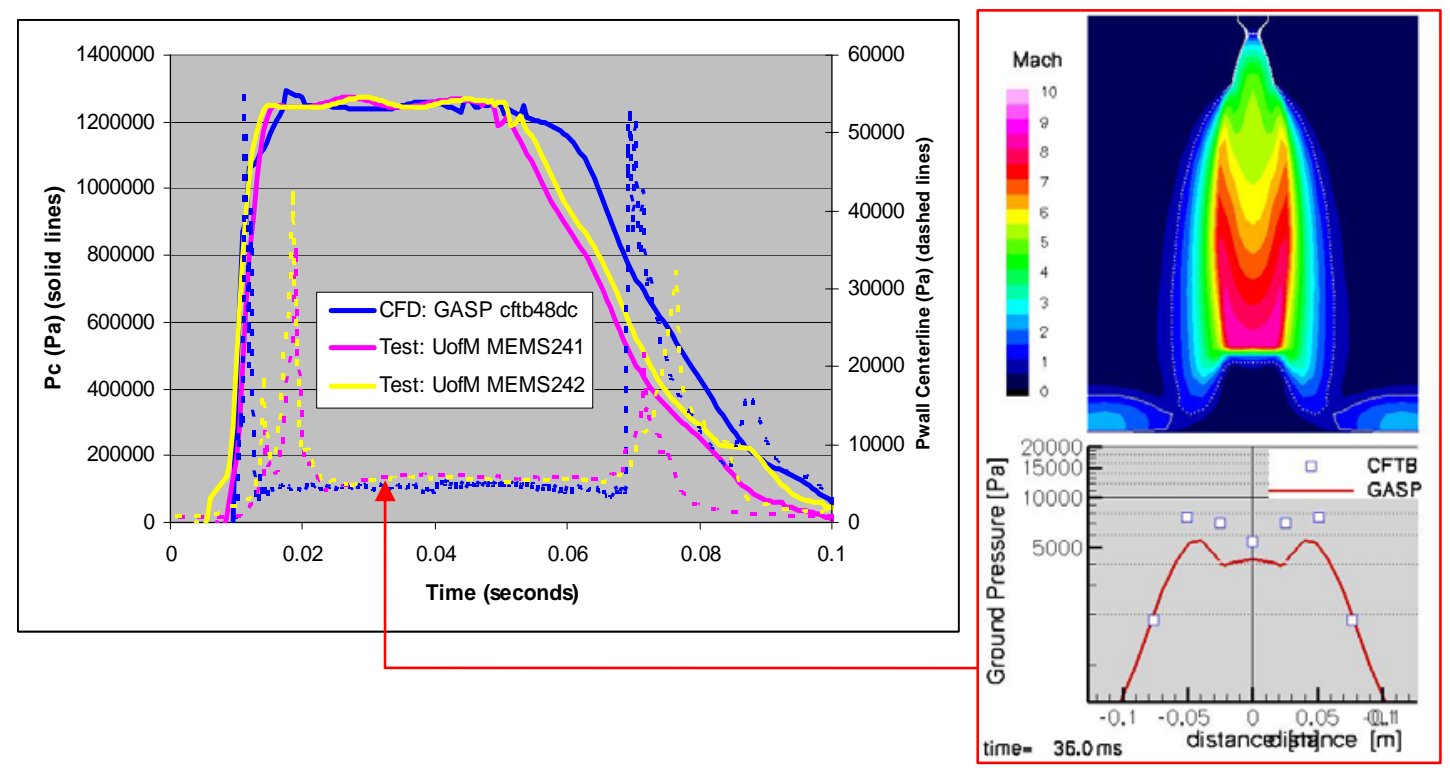

Figure 6. GASP CFD vs. experimental cold flow testing comparison at $0.5 \mathrm{~m}$ [7].

\section{Flow Structure Analyses}

Preliminary shadowgraph images confirmed that our laboratory system produces the desired supersonic plumes and has the flow visualization capabilities necessary for studying the dynamics of the impingement of pulsed rocket plumes with the ground. Preliminary shadowgraph images are comparable with results from CFD simulations (Figure 5). It shows that the jet is moderately under-expanded with an expansion ratio of approximately 4.2. The expansion ratio is determined by the ratio of ambient pressure to nozzle exit pressure. According to one-dimensional nozzle theory, the Phoenix nozzle exit Mach number is 4.5 and the exit pressure is $2.8 \mathrm{kPa}$ for both the sub-scale 
cold flow test model and full-scale TDE. The diameter of the barrel shock can be seen, but the fine oblique shock structures are not visible with our current approach.

The plume is collimated (Figure 7a). This occurs because the Mars back pressure (ambient pressure) is relatively large. This also contributes to an increase in the ground pressure and as a result Mars landings have the potential to produce more intense ground erosions than for lunar landings. The thruster plume of a lunar spacecraft during terminal descent has a larger diameter and expansion ratio because of the extremely low back pressure (the near vacuum lunar atmosphere) [8]. These plumes are more dissipative and produce smaller impingement pressure.

After the jet impinges on the surface, it expands in a radial fashion at speeds of Mach 2.1 (Figure 7). This highspeed wall jet causes significant increase in the shear stress at the surface that can lead to viscous ground erosion.
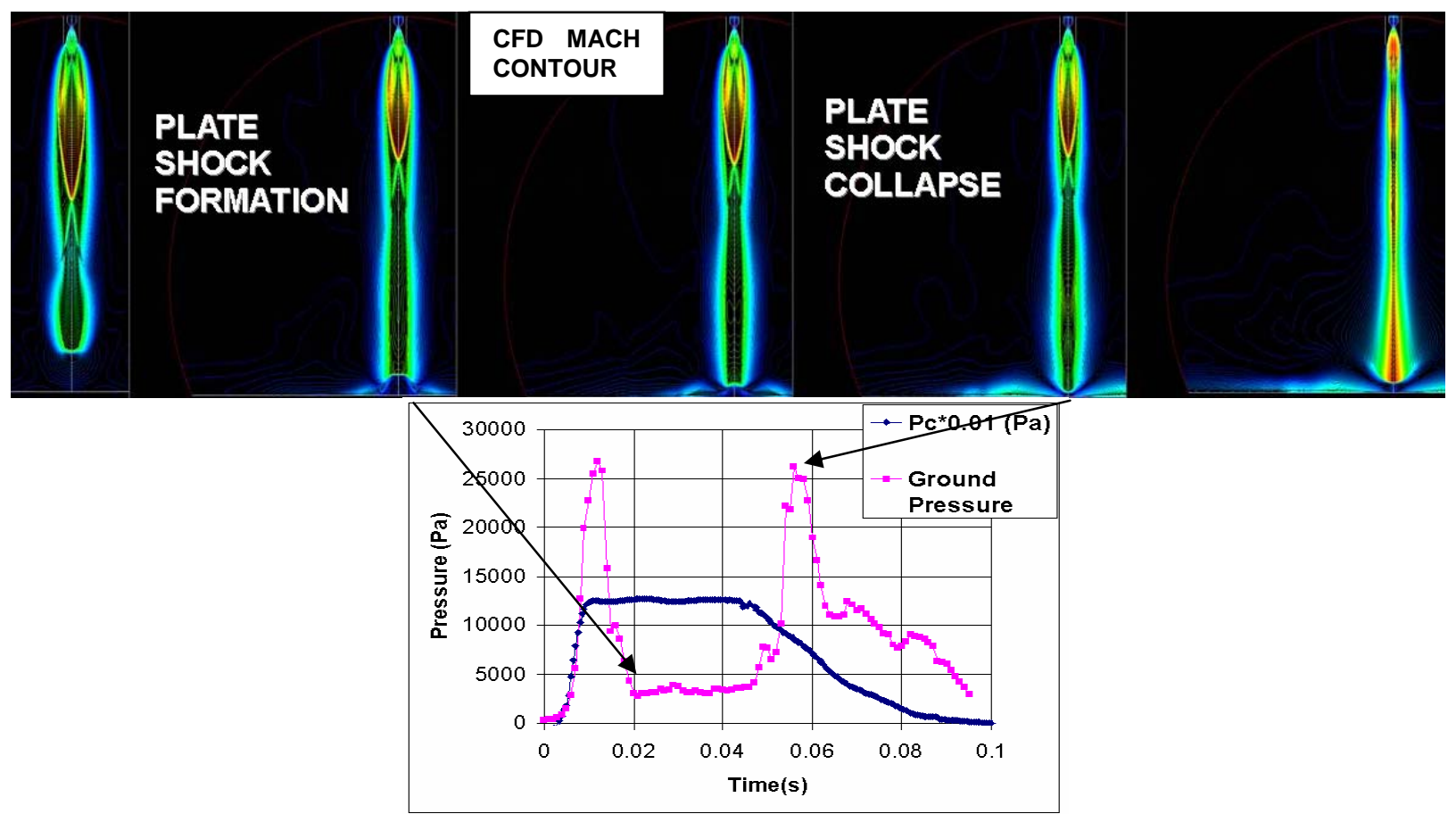

Figure 7a. Numerical simulations of the plate shock formation/ collapse process for cold flow tests at $1.25 \mathrm{~m}$.

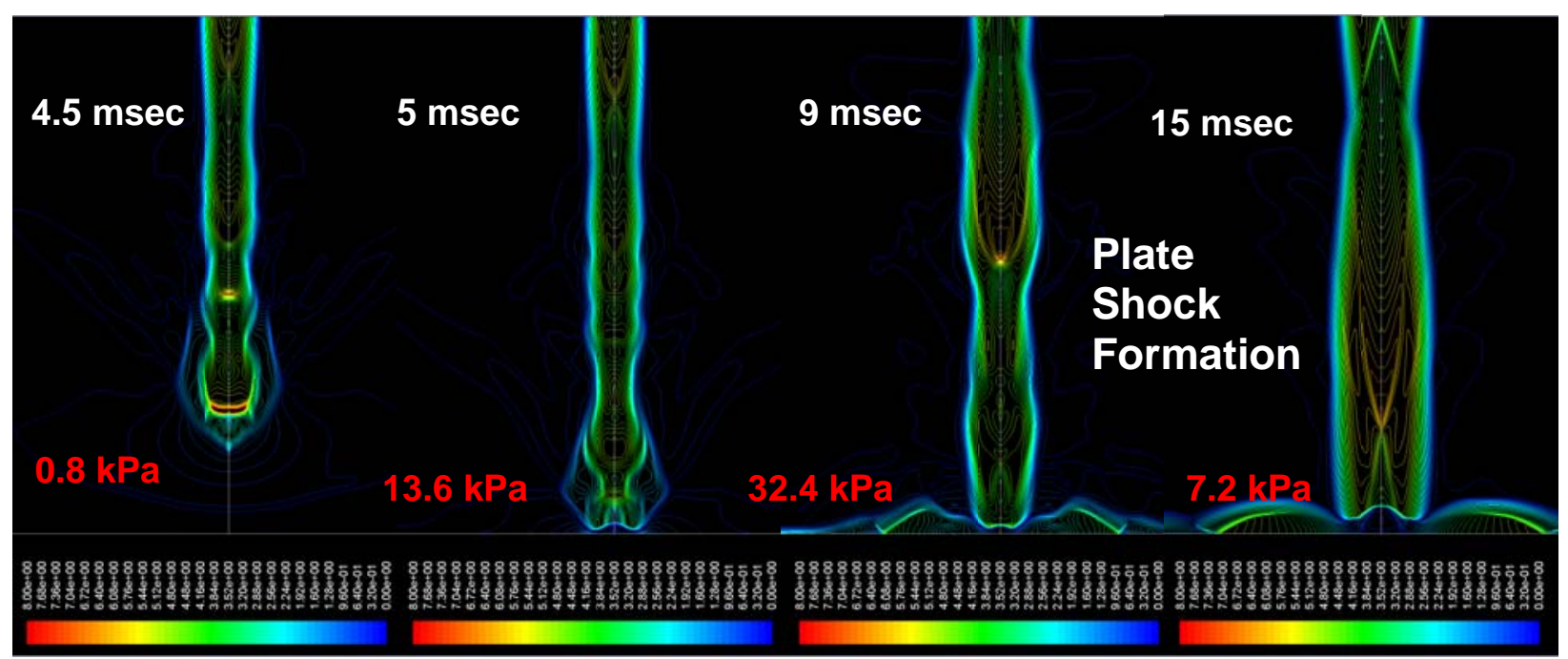

Figure 7b. Numerical simulations of the plate shock formation process for cold flow tests at $1.25 \mathrm{~m}$ (preliminary). 

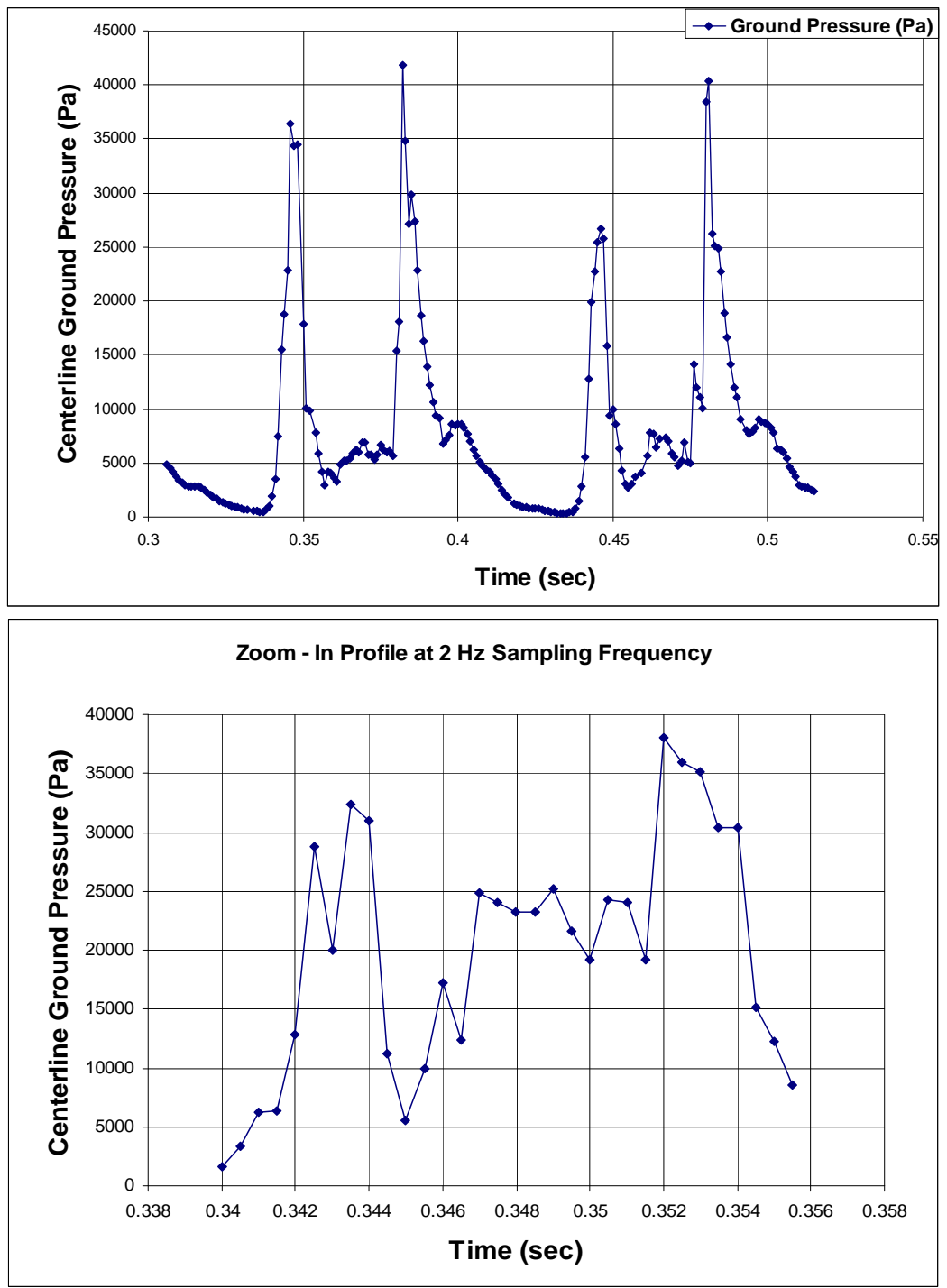

Figure 8a. Numerical simulations of centerline ground pressure vs. time during two pulse cycles-preliminary Figure 8b. Numerical simulations of centerline ground pressure during the engine startup phase-preliminary

\section{Repeatability and Instabilities}

Numerical simulations show that repeatability in the ground pressure profile can be seen for each pulse width. The two cycles displayed in Figure 8a show good agreement with each other. The discrepancy between the two cycles is predominantly due to instabilities within the plate shock. The fluctuations in the plate shock during startup, quasi-steady and shutdown phases lead to the high variability in the ground pressure results. These above numerical results (Figure 8a) were obtained with a sampling frequency of $1 \mathrm{~Hz}$ as opposed to Figure 7a which was sampled at $0.5 \mathrm{~Hz}$. Unfortunately, computational power limits our sampling frequency. The repeatability was also confirmed with our experimental cold flow test results (Figure 4b). As described earlier during the startup phase of the motor pulse width, there is significant variability in the ground pressure, because the formation of a stable plate shock is developed due to weak reflected shock instabilities. This can be seen by numerical simulation sampling frequency of $2 \mathrm{~Hz}$ (Figure 8b). The plate shock moves normal to the surface, and the smaller the standoff distance, the larger the ground pressure and vice versa. 


\section{Conclusions and Plans for the Future}

CFTB and CFD data that match the characteristics of the Phoenix MR107 motor show ground shocks with a frequency of $20 \mathrm{~Hz}$ and maximum average pressure amplitudes of $35 \mathrm{kPa}$ superimposed on a "quasi-steady" pressure perturbation of $5.0 \mathrm{kPa}$ at touchdown altitudes. These preliminary experimental and computational data show good agreement with each other. The large pressure amplitudes are strongly correlated to the plate shock formation and collapse processes of an under-expanded jet. These events have been observed to take place between altitudes of $0.53 \mathrm{~m}$ and $1.80 \mathrm{~m}$, but further studies at higher altitudes are still necessary. The ground shock perturbations may cause soil liquefaction and gas-soil bursting which could lead to large lateral ground erosion and an increase in the amount of dust lifting [11].

Once we complete the studies of pulsed supersonic jet impingement, we plan to study the effects of pulsed thruster plumes on simulated Martian and lunar soils at NASA-Ames Planetary Aeolian Laboratory (PAL) [5]. These tests are planned for September of 2007. With the technical support from JPL, NASA Ames and SETI, we have successfully completed the design and fabrication of the plume-soil experimental testbed. Preliminary measurements using this system at Earth ambient pressures were done with our diagnostic systems.

The main goal of this second phase is to study the effects of transient perturbations on "cratering" and dust lifting due to pulsed rocket plume impingement with the ground. An important concern is that soil liquefaction may occur because of high pressure ground disturbances caused by the pulsed underexpanded jets. Ground shock vibrations caused by these large pressure perturbations may disrupt the soil and reduce particle-to-particle cohesive forces. This decreases the bearing capacity of the soil and eventually may lead to ground failure and crater formation [9]. The extent of ground failure is dependent on non-linear interactions between the jet plume and the ground as well as the soil's physical properties [11]. We have matched the pressure footprint and the exhaust flow field with full-scale TDE, but non-dimensional analysis is necessary to study the dynamics of plume-soil interactions. The nondimensional parameters that need to be similar in both the CFTB and the full scale models are: hypersonic similarity term, expansion ratio, soil frictional threshold velocity, density ratio of test gas to soil, and Froude number. This analysis will allow us to build simple scaling models of soil excavation and dust lifting due to pulsed rocket plumes.

\section{Appendix}

\section{Transient Pulsed Jet Firing-10 Hz- Cold Flow}

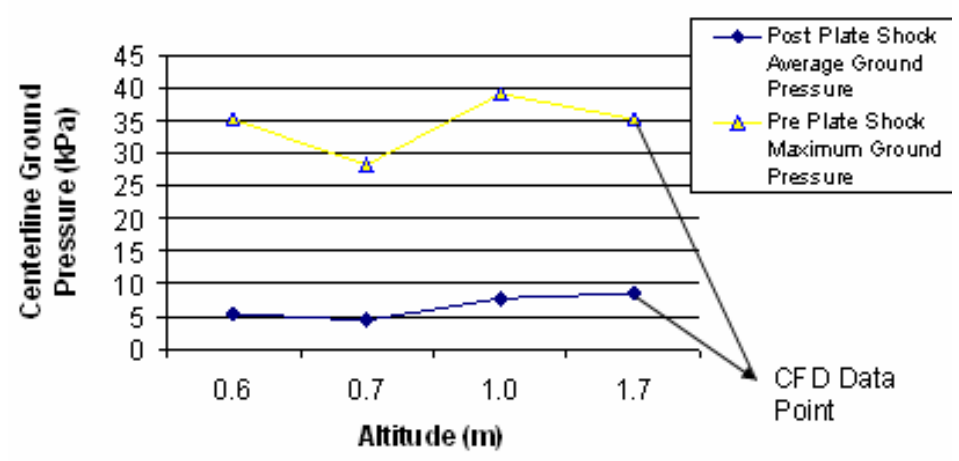

Figure 9. Ground pressure vs. altitude profiles - pulsed cold flow tests (preliminary). 


\section{Acknowledgments}

Peter G. Huseman (Orion Aerosciences - LM) and Douglas S. Gulick (Orion Aerosciences - LM) have been instrumental in this investigation. The authors also appreciate the support provided by other Phoenix Mars Scout Team members, and in particular Peter Smith (UA), Doug Adams (JPL), John Marshall (SETI), Erik Bailey (JPL), Leslie Tamppari (JPL), Tim Priser (LM) and Robert Shotwell (JPL). This research has been partially supported by the NASA GSRP grant NNX06AH56H.

\section{References}

${ }^{1}$ Alvi FS and Iyer KG "Mean and Unsteady Flowfield Properties of Supersonic Impinging Jets with Lift Plates". $5^{\text {th }}$ AIAA/CEAS Aeroacoustics Conference, AIAA 99-1829, Bellevue, WA, 1999.

${ }^{2}$ Clark LV, "Bipropellant and monopropellant rocket exhaust impingement effects on a flat plate at simulated Martian pressures. NASA Langley Working Paper, LWP - 720, 1969.

${ }^{3}$ Romine GL, Reisert TD et.al. "Site alteration effects from rocket exhaust impingement during a simulated Viking Mars landing: Nozzle development and physical site alteration”. NASA Contractor Report NASA CR-2252. Denver CO 1973.

${ }^{4}$ Grover MR, Priser TA, et.al. Phoenix EDL Critical Design Review Report. NASA-JPL PHX EDL CDR Meeting, Pasadena CA 2005.

${ }^{5}$ Mehta M, Renno NO, Gulick DS, Huseman, PG. Plume-Soil Interaction Progress Report.

Plume-Soil Working Group (PSWG) PHX EDL TIM. Lockheed Martin, Denver CO 2005.

${ }^{6}$ Huseman PG, Bomba, J. "CFD Analysis of Terminal Descent Plume Impingement for Mars Landers." $34^{\text {th }}$ AIAA Thermophysics Conference AIAA 2000-2501, Denver, CO 2000.

${ }^{7}$ Gulick DS, Mehta M, Huseman PG, Renno NO. Plume-Soil Interaction Study Presentation. Sub-Phase Terminal Descent PHX EDL TIM. Lockheed Martin, Denver, CO 2006.

${ }^{8}$ Powell A, Henderson B. "On the Tones of Round Choked Jets Impinging on Normal Flat Plates" AIAA $13^{\text {th }}$ Aeroacoustic Conference AIAA 90-3985, Talahasee FL 1990.

${ }^{9}$ Lamont PJ, Hunt BL. "The Impingement of Underexpanded Axisymetrical Jets on Perpendicular and Inclined Flat plates”. Journal of Fluid Mechanics, Vol. 100, 1980. pp. 471.

${ }^{10}$ Grossman RL. "Characteristics of Particles Blown by Exhaust Jet Impingement on a Lunar Surface". Summer Meeting AIAA 63-199. Los Angeles, CA 1963.

${ }^{11}$ Vitton S, et.al. Liquefaction and Flow Failure During Seismic Exploration. Journal of Geotechnical Engineering. Vol. 116, no. 12, pp. 1881-1899. 1990

${ }^{12}$ Aerosoft Inc. GASP Version 4.0

${ }^{13}$ FLUENT Inc., Fluent Version 6.2.16, Gambit Version 2.3. 